An audit comparing published standards to local patient education on steroid sick day rules was undertaken in 2015 , prompted by two local mortality cases related to adrenal insufficiency. Questionnaires were distributed to families for baseline data collection. Subsequent interventions included introducing 'red flags' on the electronic patient record and introducing age-banded refresher teaching sessions for families, run by staff, with patient/carer peer support opportunities.

Aims To evaluate the impact of the teaching intervention on carer knowledge and confidence around IM HC injections.

Methods Questionnaires were then redistributed to families four years later (2019). Questionnaires involved binary responses for factual questions, five-point Likert scale for opinion-based questions, and a free-text box for further comments.

Results Response rate was 94\% (49/52) in the first audit cycle (C1), where questionnaires were distributed after clinic appointments. Response rate was 36\% (39/109) in the second audit cycle (C2), with mail distribution, perhaps reflecting this change.

With particular focus on the IM HC injection, in C1, $53 \%(\mathrm{n}=26)$ received written information on IM HC injections, increasing to $69 \%(\mathrm{n}=26)$ in C2. Similarly, $61 \%$ $(n=30)$ were 'very confident' or 'somewhat confident' in C1 about when to give IM HC injection, increasing to $72 \%$ $(n=28)$ in C2. Respondents recalled demonstration on IM HC administration in 90\% $(n=44)$ in C1 and 92\% $(n=35)$ in C2. In C1 46\% $(n=22)$ were 'very confident' or 'somewhat confident' with how to give IM HC, increasing to $63 \%$ $(n=24)$ in C2.

Families have given very positive feedback, and value the opportunity to engage in peer support. There have been no further deaths in our region over the last 4 years relating to adrenal insufficiency.

Conclusion Following introduction of the refresher teaching sessions, there is a significant increase in confidence and knowledge on administration of the potentially lifesaving IM HC injection. This reflects the positive impact of a patient centred quality improvement initiative. Further work will be done to integrate these sessions into standard care.

\section{G419(P) AN OBSERVATIONAL STUDY TO EVALUATE THE CLINICAL PROFILE OF INFANTS OF DIABETIC MOTHER \& TO ASSESS THE OUTCOME IN THE PERINATAL PERIOD}

S Naidu, S Prashant. Paediatrics, Index Medical College, Indore, India

10.1136/archdischild-2020-rcpch.361

Aims

- To study clinical profile of newborns of diabetic mother.

- To assess the outcome in the perinatal period.

Material and Methods

- All infant of diabetic mothers are included in this study.

- The glycemic status assessed by estimation of FBS \& postprandial blood glucose level. Normal level should be (fasting glucose $\leq 95 \mathrm{mg} / \mathrm{dL}, 1$-hour postprandial glucose $\leq 140 \mathrm{mg} / \mathrm{dL}$, and 2-hour postprandial glucose $\leq 120$.

- Mothers antenatal history to include: socioeconomic status, family history of diabetes, previous history of macrosomic infants, usg findings.
- Mothers hba1c levels should be checked at 1st trimester for pregestational diabetes or at diagnosis of gestational diabetes.

Results

\begin{tabular}{lll}
\hline SEX distribution & & \\
\hline male & 15 cases & $72 \%$ \\
female & 10 cases & $28 \%$ \\
\hline
\end{tabular}

\begin{tabular}{lll}
\hline Birth weight & & \\
\hline SGA & 4 cases & $16 \%$ \\
AGA & 12 & $48 \%$ \\
LGA & 8 & $32 \%$ \\
\hline
\end{tabular}

IUD- $1(4 \%)$

\begin{tabular}{lll}
\hline HbA1C & & \\
\hline$>6.5$ & 9 mothers & $36 \%$ \\
$>7$ & 6 & $24 \%$ \\
$>7.5$ & 4 & $16 \%$ \\
\hline
\end{tabular}

Metabolic complications

\begin{tabular}{lll}
\hline HYPOGLYCEMIA & 12 cases & $48 \%$ \\
HYPERBILIRUBINEMIA & 10 & $40 \%$ \\
HYPOCALCEMIA & 8 & $32 \%$ \\
POLYCYTHEMIA & 4 & $16 \%$ \\
RESPIRATORY & 8 & $32 \%$ \\
DISTRESS & &
\end{tabular}

\begin{tabular}{lll}
\hline Cardiac complications & & \\
\hline SEPTAL HYPERTROPHY & 4 cases & $16 \%$ \\
PDA & 3 & $12 \%$ \\
VSD & 1 & $4 \%$ \\
ASD & 1 & $4 \%$ \\
\hline
\end{tabular}
$(8 \%)$

Other anomalies: Cleft palate- 1(4\%), Undescended testis- 2

Conclusion $48 \%$ of infants had hypoglycemia therefore strict glycemic control is required after birth of newborn of diabetic mother, onset of hypoglycemia is high typically in the first few hours after birth \& last for 2-3 days.severe prolong sympomatic hypoglycemia can result in permanent neurologic injury.

Since the incidence of cardiac and structural defects is high in IDM they should be metabolic screening for hypocalcemia, polycythemia, hyperbilirubinemia, \& echo is advised to rule out cardiac defects. Infants should be observed strictly for hypoglycemia and any respiratory distress. 